\title{
A review on various Medical Image Compression methods
}

\author{
Ms. Shrutika S. Sawant ${ }^{1}$, Dr. N. V. Dharwadkar ${ }^{2}$, Mr. Subodh. S. Ingaleshwar ${ }^{3}$ \\ Asst. Prof., Dept. of E\&TC, RIT, Sakharale, MH, India ${ }^{1,3}$ \\ Asso. Prof., Dept. of E\&TC, RIT, Sakharale, MH, India ${ }^{2}$
}

\begin{abstract}
This review paper is based on medical image compression using various image compression techniques. In this paper comparative analysis of different compression techniques and their applications in the rising fields of medical science such as e-health, telemedicine, teleradiology has been carried out. There has been abundant research work carried on medical image compression. Even though, the medical image compression has a intense scope in future but it also has lot of difficulties and challenges to achieve requirements of the medical field.
\end{abstract}

Keywords: Medical image compression, lossy compression, 3D images, SPHIT.

\section{INTRODUCTION}

Medical images are very vital for correct diagnostics and treatment. However, digital imaging generates large amounts of data which need to be compressed, without loss of significant information, to save storage space and allow speedy transfer. Different image modalities are employed for experiments in which X-rays, MRI and CT scans [1].

Compression and analysis of medical images is an important field of Biomedical Engineering. Analysis of medical images and compression of data are rapidly developing field with rapid growing applications in the healthcare services e.g. e-health, telemedicine, teleradiology, teleconsultation, [1, 2]. This has made a drastic change in the health care systems but also it has brought the idea of tele-consultation and telemedicine a reality.

\section{MEDICAL IMAGE COMPRESSION}

Medical image compression (MIC) and its analysis may even be more useful and can play an important role for the diagnosis in the telemedicine with more sophisticated and complicated images through consultation of experts [1]. The part useful for diagnosis of the image may be highlighted with more contrast and resolution for the feature extraction.

In telemedicine, it is important that image should be compressed and sent via resource constraint network, while in robotic surgery, high definition videos are required for streaming with zero tolerance error [3][4][5]. However, even with such advancement in medical science, there is a gap between the medical science and technologies available to support it with an anticipated goal. It is very important that while performing compression on the medical images, the effectiveness of resolution as well as perceptual quality be restored. It is also known that compression is also accompanied by certain loss of significant information if the data are massive and channel capacity is highly limited for transmission purpose. Another difficult in the area of medical image compression is that all there are various types of bio-medical images (x-ray, CT, MRI etc) and compression ratio applicable for all these bio-medical images highly differs from each other. Therefore, it can be seen that medical image compression is an important research issue regarding the degree of compression and the preservance of the relevant information.

\section{LITERATURE SURVEY}

There are many techniques or methods applied on medical image compression. There are main two methods of compression: Lossy \& Lossless. In lossless compression techniques, diagnosis is accurate only when compression techniques preserve all the significant and important information essential for further action. Lossy compression techniques, on the other hand, are more efficient in terms of storage and transmission needs but there is no guarantee that they can preserve all the characteristics needed in medical image processing, analysis and diagnosis.

"An overview of JPEG-2000," in Proc. IEEE Data Compress. Conf., Mar. 2000.

M. W. Marcellin, M. J. Gormish, A. Bilgin, and M. P. Boliek have proposed overview of JPEG-2000. JPEG 2000 is the state-of the art image compression DICOM standard for storage and transmission of medical images [6]. By use of another DICOM standard, which is called JPIP, the cost and feasibility of accessing large image data sets over low or various bandwidths are addressed, to enable efficient streaming of the JPEG 2000 compressed image data.

"The K-SVD: An algorithm for designing of
overcomplete
dictionaries for representation,'IEEE Trans. Signal Process., 2006.

M. Aharon [8] introduced the K-SVD rule for training of dictionaries. This rule is flexible and works in association with any pursuit rule. As such, once forced to figure with one atom per signal, it trains a dictionary for the gainshape VQ. The K-SVD is very economical, as a result of an efficient sparse writing and a Gauss-Seidel-like accelerated wordbook update technique. The algorithm's 
INTERNATIONAL JOURNAL OF INNOVATIVE RESEARCH IN ELECTRICAL, ELECTRONICS, INSTRUMENTATION AND CONTROL ENGINEERING Vol. 4, Issue 1, January 2016

steps square measure coherent with operating towards the step-down of a transparent overall objective perform.

"A lossy 3D wavelet transform for high-quality compression of medical video", The Journal of Systems and Software, 2009

Gregorio Bernabe, Jose M. Garcia and Jose Gonzalez has proposed new technique of 3D wavelet transform [9] for medical videos. For encoding the medical videos a lossy compression technique was proposed which was based on the 3D fast wavelet transform especially. The algorithm have examined and evaluated using various parameters regarding the encoding process, the counts of the times that the wavelet function is used, usage of various wavelets features, selection of the threshold value, also selection of the quantization methods and the entropy encoder. As a result a good compression ratio and a good quality of the reconstructed video have achieved. Also when this scheme is compared with MPEG-2 and EZW, it gives better compression ratios for the same Peak Signal to Noise Ratio value.

"Medical image compression using DCT-based sub band decomposition and modified SPIHT data organization", International journal of medical informatics, 2007

Yen-Yu Chen [10] has designed \& implemented a new medical image compression technique based on subband decomposition called DCT and modified SPIHT (set partitioning in hierarchical trees) data organization. Discrete Cosine Transform (DCT) was used for the subband decomposition. The detailed features of an image were stored in the translation function. In this method, high-frequency sub bands are used in good number for reduction of the redundancy by using the algorithm with modified SPIHT. Experimental results showed that the quality of the reconstructed medical image has been increased by the peak signal-to-noise ratio (PSNR) value.

"3-D medical image compression using 3-D wavelet coders", Elsevier on Digital Image Processing, 2013

B. Ramakrishnan, N. Sriraam [11] has developed a new image compression technique in the field on medical images called internet transmission of DICOM (Digital Imaging and Communications in Medicine) with effective bandwidth utilization. For the progressive transmission of DICOM images a wavelet based encoder called SPIHT (set partitioning in hierarchical trees) has been used.

"Image compression using SPIHT with modified spatial orientation trees", International Conference on Information and Communication Technologies (ICICT 2014), published in ScienceDirect.

For improving compression efficiencies for monochrome and color images, Rema N.R. [12] has proposed a new means of reordering spatial orientation tree of SPIHT. Reordering ensures that SPIHT algorithm codes more important information in the initial bits. List of insignificant pixels and sets are initialized with less number of coefficients compared to conventional SPIHT for monochrome images. For color images an altered parent offspring relationship and an extra level of wavelet decomposition on chrominance planes were performed. So this algorithm can be used in small mobile devices which are having inadequate memory and processing capability when they are compressing an image for transmission over any network.

"Fast and Efficient Lossless Image Compression Based on CUDA Parallel Wavelet Tree Encoding" IEEE, 2014

Jingqi Ao, Sunanda Mitra, Brian Nutter [13] has proposed a novel adaptation of Computation Unified Device Architecture (CUDA) to a wavelet tree based image compression algorithm. For parallelization and efficiency, both transform phase and encoding phase of the compression algorithm have been redesigned. This algorithm works well than the lossless JPEG-XR algorithm, with good compression ratios. The performance of this algorithm faster than the lossless JPEG-XR algorithm. PCWT can achieve six times faster encoding speed than a popular JPEG-XR implementation, if a parallel lossless wavelet transform and an MQD based coefficient encoding algorithm is carefully designed. Because most of the parallel steps do not have any hardware specification limitations, scalability of PCWT is high.

"Wavelet transforms and polynomial approximation model for lossless medical image compression" International Journal of Advanced Research in Computer Science and Software Engineering, 2014

Dr.Ghadah AJ-Khafaji [14] had proposed simple lossless image compression method for compressing medical image, based on efficiently exploiting the polynomial representation model along with wavelet transform. The result shows that high compression ratio has achieved with fully noisy reconstruction, its flexibility of use leads to smaller amount of compressed information required.

"Resolution Scalable Coding and Region of Interest Access with Three-Dimensional SBHP Algorithm", Third International symposium on 3D Data Processing, Jun 2006.

The Subband Block Hierarchial Partitioning (SBHP) algorithm [15] is extended to three dimension modified algorithm, and applied to every code block independently. The algorithm, 3D-SBHP, efficiently encodes 3D image data by the exploitation of the dependencies in all dimensions, while enabling progressive SNR and resolution decompression and Region-of-Interest (ROI) access from the same bit stream. The code-block selection method by which random access decoding can be achieved is outlined. The 2-D SBHP algorithm is a SPECK variant which was originally designed as a low complexity alternative to JPEG2000. In 3-D SBHP, each sub-band is partitioned into same sized code-blocks. Then 3-D SBHP is applied to every block separately which generates a highly scalable bitstream for each code-block. But in this scheme the background information is only decoded after the VOI is fully decoded, which prevents observing the position of the VOI within the original 3-D image.

\section{RESEARCH GAP}

From the review, it can be seen that adoption of certain standards like JPEG lossless compression is quite high as 
well as lossy compression techniques too [16]. For the techniques that implements discrete wavelet transforms, the perceptual quality of the medical image is found to be considerably high. The lossy compression technique seen on majority of chest radiological images, although found in acceptable compression ratio, but they fail to achieve significant minimization in the identification capability [17]. Hence, a research gap is found that efficient technique for compression by emphasizing the region of diagnostic importance and discarding the other regions surfaces up. Working toward such issues may retain the clinical importance of the data carried by the region of diagnostic significance and will be helpful in future even for telemedicine.

\section{CONCLUSION}

Even though there exists abundant research work on medical image compression considering lossy and lossless types, but the need of medical images to be compressed efficiently with optimal compression ratio is yet a question mark. Also it has been found from previous research work that most of the work was intended to the 2D medical images. So, there is need to develop effective compression algorithm for 3D medical images.

\section{REFERENCES}

[1] J. S. Duncan, and N. Ayache, "Medical Image Analysis, Progress over two Decades and the Challenges Ahead," IEEE Transactions on Pattern Analysis and Machine Intelligence, Vol. 22, No.1, pp. 85-106, 2000

[2] Gonzalez, Woods and Eddies. "Digital Image Processing Using MATLAB”, 2009.

[3] Feng, D., Cai, W., and Fulton, R .2002. Dynamic image data compression in the spatial and temporal domains: clinical issues and assessment. IEEE Transaction on Information Technology in Biomedicine.Vol.6, pp.262268, Issue.4

[4] Portoni, L., Combi, C., Pinciroli, F.2002. User-Oriented Views in Health Care Information Systems. IEEE Transactions on Biomedical Engineering. Vol. 49, No. 12

[5] Al-Shaykh, O.K., and Mersereau, R.M.1996. Lossy compression of noisy cardiac image sequences. IEEE Proc. of the Data Compression Conf., pp.43-52, Vol. 31

[6] S.S. Maniccam and N.G. Bourbakis, "Scan based Lossless Image Compression and Encryption", In Information Intelligence and Systems, International Conference on, pp- 490-499, 1999.

[7] Y.Liu and W.A.Pearlman," Resolution Scalable Coding and Region of Interest Access with Three-Dimensional SBHP Algorithm", Third International symposium on 3D Data Processing, Jun 2006.

[8] M. Aharon, M. Elad, and A. M. Bruckstein, "The K-SVD: An algorithm for designing of overcomplete dictionaries for sparse representation,'IEEE Trans. Signal Process., vol. 54, pp. 43114322, 2006.

[9] Gregorio Bernab , Jose M. García , José González ,"A lossy 3D wavelet transform for high-quality compression of medical video", The Journal of Systems and Software, Vol. 82, pp.526-534,2009

[10] Yen-Yu Chen, "Medical image compression using DCT-based sub band decomposition and modified SPIHT data organization", International journal of medical informatics, Vol.76, pp. 717-725, 2007

[11] N. Sriraam, R. Shyamsunder." 3-D medical image compression using 3-D wavelet coders", Elsevier on Digital Image Processing, Vol.21, pp.100-109, 2013

[12] Rema N.R. "Image compression using SPIHT with modified spatial orientation trees", International Conference on Information and Communication Technologies (ICICT 2014), published in ScienceDirect.

[13] Jingqi Ao, Sunanda Mitra, Brian Nutter "Fast and Efficient Lossless Image Compression Based on CUDA Parallel Wavelet Tree Encoding" IEEE, 2014
14] Ghadah AJ-Khafaji,"Wavelet transforms and polynomial approximation model for lossless medical image compression", International Journal of Advanced Research in Computer Science and Software Engineering, vol.4, 2014

[15] "Resolution Scalable Coding and Region of Interest Access with Three-Dimensional SBHP Algorithm", Third International symposium on 3D Data Processing, Jun 2006

[16] Grgic, S., Grgic, M., Cihlar, B. Z.2001. Performance Analysis of Image Compression Using Wavelets. IEEE Transactions on Industrial Electronics, Vol. 48, No. 3

[17] Wong, S.1995. Radiologic Image Compression-A Review, IEEE Trans. on Medical Imaging. Vol. 83, No. 2 